\title{
Estudo de correlação da anemia ferropriva, deficiência de ferro, carência nutricional e fatores associados: Revisão de literatura
}

Correlation study of iron deficiency anemia, iron deficiency, nutritional deficiency and associated

\author{
factors: Literature review
}

Estudio de correlación de anemia por deficiencia de hierro, deficiencia de hierro, deficiencia nutricional y factores asociados: Revisión de la literatura

Recebido: 05/07/2021 | Revisado: 10/07/2021 | Aceito: 17/07/2021 | Publicado: 26/07/2021

\author{
Isabele Felix Mortari \\ ORCID: https://orcid.org/0000-0002-6521-8137 \\ Escola Superior da Amazônia, Brasil \\ E-mail: isabelefelixmortari@gmail.com \\ Murilo Tavares Amorim \\ ORCID: https://orcid.org/0000-0002-9769-2183 \\ Universidade Federal do Pará, Brasil \\ E-mail: muriloamorimbio@gmail.com \\ Michele Amaral da Silveira \\ ORCID: https://orcid.org/0000-0001-5119-7526 \\ Escola Superior da Amazônia, Brasil \\ E-mail: mi_biom@yahoo.com.br
}

\begin{abstract}
Resumo
O objetivo desse estudo foi realizar uma revisão de literatura sobre a anemia ferropriva, com ênfase na correlação entre a carência nutricional e fatores relacionados. Utilizando as bases de dados Scielo, PubMed, Google Acadêmico e BVS, nos idiomas português, inglês e espanhol, utilizando dos seguintes critérios de inclusão: publicações feitas em janeiro de 2010 até 2021, que correspondem aos objetivos propostos, destacando o diagnóstico, o tratamento e a prevenção da anemia ferropriva e os seguintes critérios de exclusão: publicados anteriores à 2010 e que não correspondem aos objetivos. Foram selecionados 19 artigos que se enquadraram aos objetivos desejados. Mediante a pesquisa realizada foi possível concluir que a anemia ferropriva é considerada a mais comum das anemias, podendo ser citada como um sério problema de Saúde Pública, com destaque principal nos países em desenvolvimento. Além disso pode ser visto que a anemia ferropriva é causada por diversos fatores que podem ser internos e externos, onde qualquer sintoma sendo ele específico ou não precisa ser analisado, é necessário que seja feito um diagnostico detalhado, que seu tratamento seja completo e acompanhado por um profissional da área e que modos de prevenção sejam passados para a sociedade e assim evitar que os indivíduos possam sofrer com essa condição.
\end{abstract}

Palavras-chave: Anemia ferropriva; Ferro; Deficiência de ferro.

\begin{abstract}
The aim of this study was to carry out a literature review on iron deficiency anemia, with an emphasis on the correlation between nutritional deficiency and related factors. Using the Scielo, PubMed, Academic Google and VHL databases, in Portuguese, English and Spanish, using the following inclusion criteria: publications made in January 2010 until 2021, which correspond to the proposed objectives, highlighting the diagnosis, treatment and the prevention of iron deficiency anemia and the following exclusion criteria: published prior to 2010 and not meeting the objectives. Nineteen articles were selected that fit the desired objectives. Through the research carried out, it was possible to conclude that iron deficiency anemia is considered the most common of anemia, and can be cited as a serious public health problem, with main emphasis in developing countries. In addition, it can be seen that iron deficiency anemia is caused by several factors that can be internal and external, where any symptom, whether specific or not needing to be analyzed, requires that a detailed diagnosis be made, that its treatment be complete and accompanied by a professional in the area and that prevention methods are passed on to society and thus prevent individuals from suffering from this condition.
\end{abstract}

Keywords: Iron deficiency anemia; Iron; Iron deficiency.

\section{Resumen}

El objetivo de este estudio fue realizar una revisión de la literatura sobre anemia ferropénica, con énfasis en la correlación entre la deficiencia nutricional y factores relacionados. Utilizando las bases de datos Scielo, PubMed, Academic Google y VHL, en portugués, inglés y español, utilizando los siguientes criterios de inclusión: publicaciones realizadas en enero de 2010 hasta 2021, que corresponden a los objetivos propuestos, destacando el diagnóstico, 
tratamiento y prevención del hierro. anemia por carencia y los siguientes criterios de exclusión: publicados antes de 2010 y no cumpliendo los objetivos. Se seleccionaron diecinueve artículos que se ajustaban a los objetivos deseados. A través de la investigación realizada, se pudo concluir que la anemia ferropénica es considerada la anemia más común y puede ser citada como un problema grave de salud pública, con mayor énfasis en los países en desarrollo. Además, se puede observar que la anemia ferropénica es causada por varios factores que pueden ser internos y externos, donde cualquier síntoma, ya sea específico o no que necesite ser analizado, requiere que se haga un diagnóstico detallado, que su tratamiento sea completo y acompañados de un profesional del área y que los métodos de prevención se transmitan a la sociedad y así evitar que las personas padezcan esta condición.

Palabras clave: La anemia por deficiencia de hierro; Planchar; Deficiencia de hierro.

\section{Introdução}

A forma mais comum de carência nutricional é a deficiência de ferro, que atinge os países desenvolvidos e os em desenvolvimento, além disso o ferro é considerado um dos elementos que faz parte da hemoglobina (Rodrigues, 2011). O ferro é absorvido no duodeno, que é a primeira parte do intestino delgado (Antunes \& Canziani, 2016) e é proveniente de diversos alimentos como: feijão, beterraba, carnes vermelhas entre outros (Brasil, 2015). Estima-se que no mundo mais ou menos dois bilhões de pessoas apresentam algum tipo de anemia e que dentre essas pessoas cerca de $27 \%$ a $50 \%$ são afetadas pela deficiência de ferro (Fisberg et al., 2018). Justino (2017) afirma que no Brasil a prevalência de anemia ferropriva em crianças de 0 a 10 anos é de $41 \%$.

As causas da anemia ferropriva (AF) podem ser: má absorção de ferro, que pode ser causada por conta de uma gastrite atrófica por exemplo, o baixo consumo de ferro na alimentação, perda aguda de sangue por consequência de traumas e perda crônica de sangue (Filho \& Barros, 2013). A AF também apresenta sintomas inespecíficos que são considerados assim pois podem estar presentes em outras anemias, sintomas como: dificuldade de aprendizagem nas crianças, fadiga generalizada, anorexia, palidez de pele e mucosas, menor disposição para o trabalho, apatia (Brasil, 2015).

Sato et al. (2018) explica que embora o diagnóstico possa ser feito por meio do exame físico e do histórico do paciente o mais assertivo é o exame laboratorial, pois ele confirmará o quadro clínico do paciente.

$\mathrm{O}$ tratamento da anemia ferropriva pode ser feito das seguintes maneiras: transfusão de hemácias que é indicada para perca contínua de sangue e instabilidade cardiovascular, terapia com ferro que inclui sais de ferro simples e compostos de ferro complexos e a ferroterapia parenteral, onde o ferro é administrado por via intravenosa (Jameson et al., 2020).

Segundo Brasil (2013) também podemos contar com a prevenção, que pode ser feita a partir da gestação com a ingestão de alimentos adequados e saudáveis que contenham ferro, no nascimento com a amamentação na primeira hora de vida e suplementação profilática de ferro para crianças prematuras.

A anemia é considerada uma condição patológica onde ocorre a diminuição da massa de hemoglobina e da massa eritrocitária. A concentração da hemoglobina é o parâmetro laboratorial mais utilizado para definir o quadro de anemia, porém sua redução não define somente a anemia, pois sua diminuição pode ocorrer em situações fisiológicas, como no segundo trimestre da gestação (SANTIS, 2019). Segundo a Organização Mundial de Saúde (BRASIL, 2015) a anemia pode ser definida como a condição na qual o conteúdo de hemoglobina no sangue está à baixo dos parâmetros normais por consequência da carência de um ou mais nutrientes essenciais. As anemias podem ser causadas por deficiência de vários nutrientes como o zinco, vitamina B12, ferro entre outras. A deficiência de ferro (anemia ferropriva), é classificada como sendo uma das carências nutricionais mais prevalentes. Jordão (2009) afirma que a anemia por deficiência de ferro é considerada a mais comum das carências nutricionais que é prevalente em mulheres e crianças, com destaque principal nos países em desenvolvimento, sendo então as anemias consideradas um sério problema de Saúde Pública.

A anemia ferropriva é uma doença nutricional que compromete o sistema imunológico, podendo prejudicar o desenvolvimento e crescimento da criança, por tanto as crianças constituem um grupo vulnerável a deficiência de ferro, pois precisam de uma demanda maior desse mineral em função da velocidade de crescimento (ANDRÉ et al., 2018). Entre os casos 
de anemia ao redor do mundo a deficiência de ferro é considerada a principal, no qual atinge 50\% da população que apresenta algum tipo de anemia, incluindo principalmente lactantes, crianças com menos de cinco anos de idade, gestantes e mulheres em idade reprodutiva (Siqueira \& Lenhard-Vidal, 2019)

Segundo Santis (2019) em uma cidade do interior do Acre foi realizado um estudo em 1.111 crianças, de 6 meses a 10 anos, que mostrou prevalência de anemia em 13,6\% das crianças, de anemia ferropriva em 10,3\% dos investigados e de deficiência de ferro em 45,4\% dos indivíduos. Lopes et al (2019) afirma que a anemia ferropriva afeta principalmente o público infantil, tal fato pode ser resultado de mudanças nos hábitos alimentares, que acompanham a transição nutricional no país. Dois estudos indicam que no Brasil ocorre a tendência do aumento da anemia em pré-escolares, nos quais a prevalência da doença passou de 35,6\% em 2016 para 46,9\% em 2018, no município de São Paulo, e de 19,3\% para 36,4\%, no Ceará. Maman (2020) cita que entre seis e 24 meses de idade se tem um grande risco de desenvolver essa condição e que 25 e 60 meses o risco é duas vezes maior. Além disso a prevalência de anemia ferropriva no Brasil é de 53\%, sendo as regiões Norte e Centro-Oeste as que possuem maior número de pessoas com a doença e que em nível mundial, até 10\% da população vem com a doença. Portanto, nota-se que a Anemia Ferropriva é uma condição que exige um acompanhamento específico que vise regular o se desenvolvimento.

\section{Metodologia}

Foi realizada uma revisão bibliográfica qualitativa, desenvolvida durante o período de agosto de 2020 a junho de 2021. Buscando publicações de janeiro de 2010 a 2021, as bases de dados que foram utilizadas são: Scientific Electronic Library Online (Scielo), PubMed, Google Acadêmico e Biblioteca Virtual em Saúde do Ministério da Saúde (BVS MS), nos idiomas português, inglês e espanhol. Para as buscas nas bases de dados foram utilizadas as seguintes palavras-chaves: anemia ferropriva, ferro e deficiência de ferro. Os critérios de inclusão foram: publicações feitas em janeiro de 2010 até 2021, como foco principal nas publicações que corresponderem aos objetivos propostos, destacando o diagnóstico, o tratamento e a prevenção da anemia ferropriva com resumos disponíveis e que poderão ser acessados na íntegra pelo meio on-line nos idiomas inglês, português ou espanhol. Foram excluídas publicações anteriores à 2010 que não corresponderam aos objetivos (Figura 1).

Figura 1: Fluxograma da metodologia.

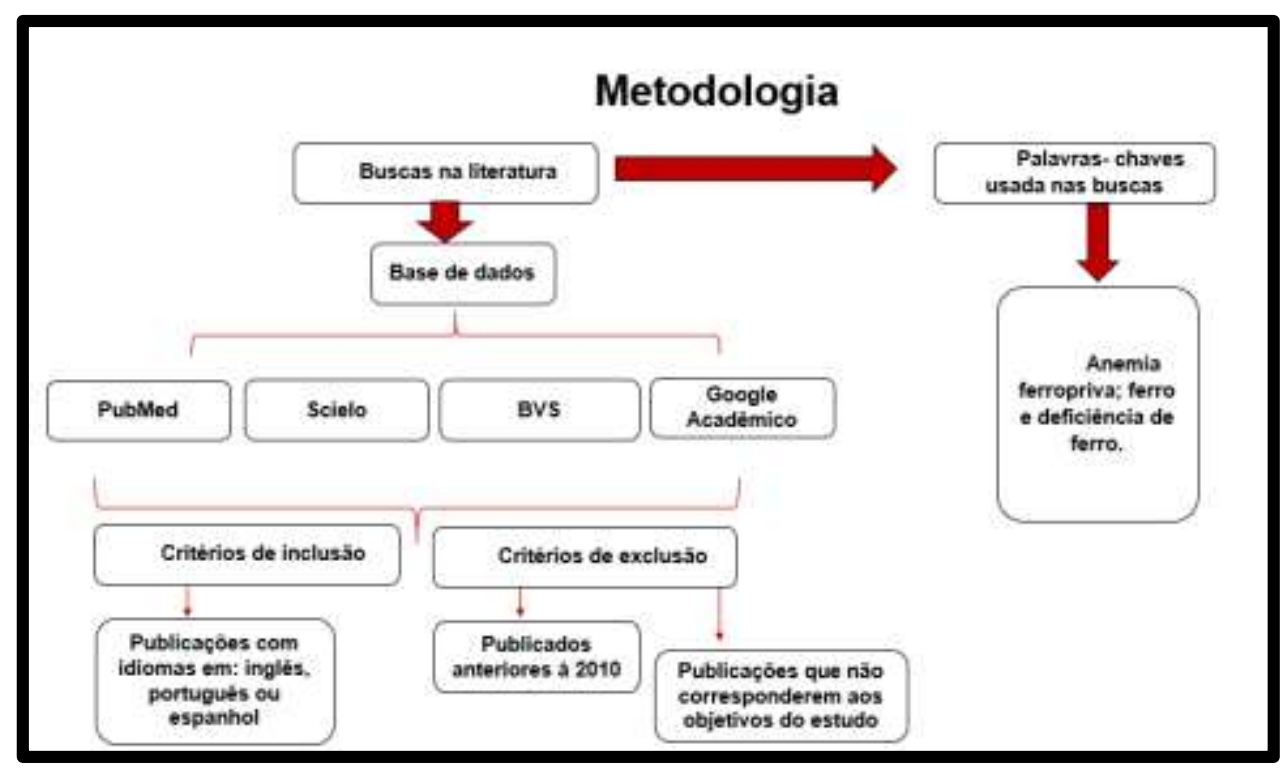

Fonte: Autores. 
Para a apreciação e síntese dos artigos selecionados para esse estudo, foi construída um quadro sinóptico contendo os seguintes aspectos: título do artigo, autor (es), base da dados, síntese e ano de publicação.

\section{Resultados}

Utilizando as bases de dados mencionadas na metodologia foram selecionados um total de 46 artigos, sendo 4 publicados em 2 bases de dados e $1 \mathrm{em} 3$ bases de dados, após a retirada dos artigos repetidos restaram 40 artigos (Figura 2), que passaram pelos critérios de inclusão e exclusão, destes, 19 foram selecionados por se enquadrarem aos objetivos desejados.

Figura 2: Artigos selecionados.

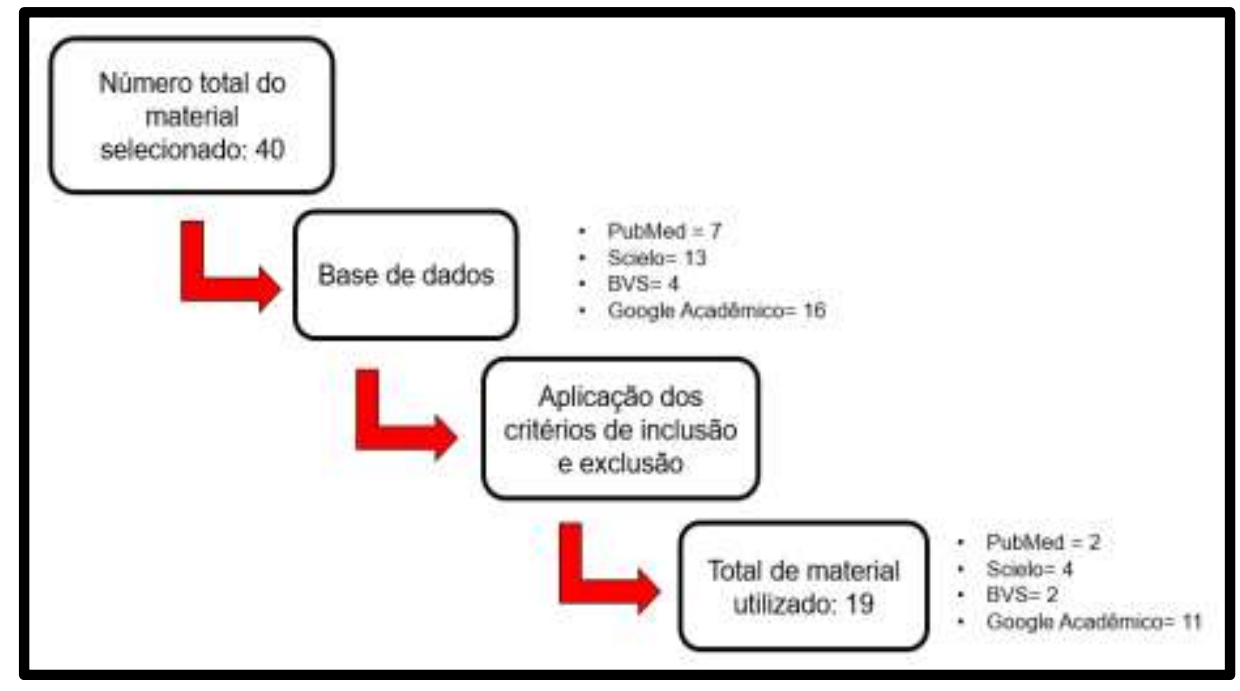

Fonte: Autores.

Os estudos foram selecionados, pois corresponderam aos objetivos designados. Os artigos que corresponderam ao primeiro objetivo que foi: classificar causas e sintomas da anemia ferropriva estão listados no Quadro 1. 
Quadro 1: Classificação das causas e sintomas da anemia ferropriva.

\begin{tabular}{|c|c|c|c|c|c|}
\hline & Título do artigo & Autor (es) & Síntese & Base de dados & Ano \\
\hline 1 & $\begin{array}{l}\text { Anemia ferropênica no } \\
\text { adulto: causas, diagnóstico } \\
\text { e tratamento }\end{array}$ & $\begin{array}{l}\text { CANÇADO, } \\
\text { Rodolfo D.; } \\
\text { CHIATTONE, } \\
\text { Carlos S. }\end{array}$ & $\begin{array}{l}\text { Compostos com ferro atualmente disponíveis, } \\
\text { o perfil de eficácia, segurança e tolerabilidade } \\
\text { desses medicamentos, e o plano terapêutico } \\
\text { ideal para o sucesso no tratamento dessa } \\
\text { doença tão comum e importante. }\end{array}$ & Scielo & 2010 \\
\hline 2 & Anemia & $\begin{array}{l}\text { BRASIL. } \\
\text { Ministério da } \\
\text { Saúde }\end{array}$ & $\begin{array}{l}\text { O ferro é um nutriente essencial para a vida e } \\
\text { atua no transporte do oxigênio para todas as } \\
\text { células do corpo e na fabricação das células } \\
\text { vermelhas do sangue. }\end{array}$ & BVS & 2015 \\
\hline 3 & $\begin{array}{l}\text { Anemia associada às } \\
\text { parasitoses intestinais de } \\
\text { pacientes atendidos em um } \\
\text { laboratório de análises } \\
\text { clínicas no município de } \\
\text { Juazeiro do Norte-CE. }\end{array}$ & $\begin{array}{l}\text { JÚNIOR, José } \\
\text { Geraldo de } \\
\text { Alencar } \\
\text { Santos et al. }\end{array}$ & $\begin{array}{l}\text { Valores de concentração de hemoglobina } \\
\text { como parâmetros hematológicos para definir } \\
\text { o diagnóstico de anemia. }\end{array}$ & $\begin{array}{c}\text { Google } \\
\text { Acadêmico }\end{array}$ & 2016 \\
\hline 4 & Iron deficiency. & $\begin{array}{l}\text { CAMASCHE } \\
\text { LLA, Clara }\end{array}$ & $\begin{array}{l}\text { Total-body (absolute) iron deficiency is } \\
\text { caused by physiologically increased iron } \\
\text { requirements in children, adolescents, young } \\
\text { and pregnant women, by reduced iron intake, } \\
\text { or by pathological defective absorption or } \\
\text { chronic blood loss }\end{array}$ & PubMed & 2019 \\
\hline 5 & $\begin{array}{l}\text { Anemia: definição, } \\
\text { epidemiologia, } \\
\text { fisiopatologia, classificação } \\
\text { e tratamento }\end{array}$ & $\begin{array}{l}\text { SANTIS, Gil } \\
\text { Cunha de }\end{array}$ & $\begin{array}{l}\text { Nesse artigo de revisão, discute-se a } \\
\text { prevalência da anemia, sua classificação, } \\
\text { fisiopatologia, os tipos mais comuns e o } \\
\text { tratamento. }\end{array}$ & $\begin{array}{c}\text { Google } \\
\text { Acadêmico }\end{array}$ & 2019 \\
\hline 6 & Anemia ferropriva & $\begin{array}{l}\text { MAMAN, } \\
\text { Maria Julia } \\
\text { Cavaler De }\end{array}$ & $\begin{array}{l}\text { As causas de deficiência de ferro e } \\
\text { consequente anemia são diversas e podem ser } \\
\text { divididas de acordo com sua origem. }\end{array}$ & $\begin{array}{c}\text { Google } \\
\text { Acadêmico }\end{array}$ & 2020 \\
\hline
\end{tabular}

Fonte: Autores.

Os artigos selecionados que correspondem ao segundo objetivo que foi: descrever importantes pontos da patogenia e do diagnóstico estão listados no Quadro 2. 
Quadro 2: Patogenia e diagnóstico.

\begin{tabular}{|c|c|c|c|c|c|}
\hline & Título do artigo & Autor (es) & Síntese & Base de dados & Ano \\
\hline 1 & $\begin{array}{l}\text { Diagnóstico laboratorial da } \\
\text { deficiência de ferro. }\end{array}$ & $\begin{array}{l}\text { GROTTO, Helena } \\
\text { ZW. }\end{array}$ & $\begin{array}{l}\text { Testes que auxiliam na investigação } \\
\text { da deprivação de ferro, com } \\
\text { algumas noções técnicas e } \\
\text { comentários sobre a interpretação } \\
\text { dos mesmos. }\end{array}$ & Scielo & 2010 \\
\hline 2 & $\begin{array}{l}\text { Utilização De Marcadores } \\
\text { Hematológicos Para } \\
\text { Diferenciação De Anemia } \\
\text { Ferropriva De Anemia De } \\
\text { Doença Crônica. }\end{array}$ & $\begin{array}{l}\text { NUNES, Deise } \\
\text { Candido; } \\
\text { BARONE, } \\
\text { Alessandra }\end{array}$ & $\begin{array}{l}\text { Importância dos marcadores } \\
\text { hematológicos para a diferenciação } \\
\text { dos tipos de anemias ferropriva e } \\
\text { crônica. }\end{array}$ & $\begin{array}{l}\text { Google } \\
\text { Acadêmico }\end{array}$ & 2013 \\
\hline 3 & Anemia e Senilidade. & $\begin{array}{l}\text { GALEIGO, } \\
\text { Fernanda Pereira } \text { et } \\
\text { al. }\end{array}$ & $\begin{array}{l}\text { Os níveis de hemoglobina utilizados } \\
\text { para identificação de anemia é }<12 \\
\text { g/dL para mulheres e }<13 \mathrm{~g} / \mathrm{dL} \text { para } \\
\text { homens, além da contagem de } \\
\text { hemácias, RDW e índices } \\
\text { hemantimétricos. }\end{array}$ & $\begin{array}{l}\text { Google } \\
\text { Acadêmico }\end{array}$ & 2014 \\
\hline 4 & $\begin{array}{l}\text { Anemia por deficiencia de } \\
\text { hierro en el lactante. }\end{array}$ & $\begin{array}{l}\text { SVARCH } \\
\text { GUERCHICOFF, } \\
\text { Eva }\end{array}$ & $\begin{array}{l}\text { El diagnóstico incluye la detección } \\
\text { de microcitosis e hipocromía en la } \\
\text { extensión de sangre periférica, la } \\
\text { determinación de las constantes } \\
\text { corpusculares. }\end{array}$ & Scielo & 2015 \\
\hline 5 & $\begin{array}{l}\text { Abordagem dos principais } \\
\text { aspectos relacionados à } \\
\text { anemia ferropriva. }\end{array}$ & $\begin{array}{l}\text { MACHADO, G. S. } \\
\quad \text { et al. }\end{array}$ & $\begin{array}{l}\text { Testes que possam auxiliar no } \\
\text { diagnóstico assim como aspectos } \\
\text { relacionados à anemia ferropriva. }\end{array}$ & $\begin{array}{c}\text { Google } \\
\text { Acadêmico }\end{array}$ & 2016 \\
\hline 6 & Anemia ferropriva & $\begin{array}{l}\text { YAMAGISHI, } \\
\text { Jessica Akemi et } \\
\text { al. }\end{array}$ & $\begin{array}{l}\text { Esta patologia é considerada um } \\
\text { grande problema de saúde pública e } \\
\text { os grupos mais susceptíveis são } \\
\text { gestantes, lactentes, adolescentes e } \\
\text { pré-escolares. }\end{array}$ & $\begin{array}{l}\text { Google } \\
\text { Acadêmico }\end{array}$ & 2017 \\
\hline 7 & $\begin{array}{l}\text { Indicadores de insegurança } \\
\text { alimentar e nutricional } \\
\text { associados à anemia } \\
\text { ferropriva em crianças } \\
\text { brasileiras: uma revisão } \\
\text { sistemática }\end{array}$ & $\begin{array}{l}\text { ANDRÉ, Hercilio } \\
\text { Paulino et al. }\end{array}$ & $\begin{array}{l}\text { Os resultados dos estudos } \\
\text { retrataram que a anemia ferropriva } \\
\text { em crianças } \\
\text { brasileiras associou-se aos } \\
\text { indicadores sociodemográficos e de } \\
\text { saúde, indicadores } \\
\text { nutricionais e econômicos. }\end{array}$ & Scielo & 2018 \\
\hline 8 & $\begin{array}{l}\text { Consenso Sobre Anemia } \\
\text { Ferropriva: Mais Que Uma } \\
\text { Doença, Uma Urgência } \\
\text { Médica }\end{array}$ & $\begin{array}{l}\text { GURMINI, } \\
\text { Jocemara et al. }\end{array}$ & $\begin{array}{l}\text { A suplementação com ferro deve ser } \\
\text { iniciada aos } 3 \text { meses de idade e } \\
\text { mantida pelo menos até o segundo } \\
\text { ano de vida, independentemente do } \\
\text { regime de aleitamento. }\end{array}$ & $\begin{array}{l}\text { Google } \\
\text { Acadêmico }\end{array}$ & 2018. \\
\hline 9 & $\begin{array}{l}\text { Diagnóstico e tratamento da } \\
\text { anemia ferropriva. }\end{array}$ & $\begin{array}{l}\text { FREIRE, Sarah } \\
\text { Torres et al. }\end{array}$ & $\begin{array}{l}\text { A partir das informações obtidas } \\
\text { neste estudo, } \\
\text { verifica-se que a anemia ferropriva } \\
\text { ainda é elevada nos } \\
\text { grupos mais vulneráveis, crianças, } \\
\text { mulheres em idade } \\
\text { reprodutiva e grávidas. }\end{array}$ & $\begin{array}{l}\text { Google } \\
\text { Acadêmico }\end{array}$ & 2020 \\
\hline
\end{tabular}

Fonte: Autores.

Os artigos do Quadro 3 estão os correspondentes ao terceiro objetivo que foi: caracterizar as formas de tratamento e prevenção. 
Quadro 3: Formas de tratamento e prevenção.

\begin{tabular}{|c|c|c|c|c|c|}
\hline & Título do artigo & Autor (es) & Síntese & Base de dados & Ano \\
\hline 1 & $\begin{array}{l}\text { Programa Nacional de } \\
\text { Suplementação de Ferro: } \\
\text { manual de condutas gerais. }\end{array}$ & $\begin{array}{l}\text { BRASIL. Ministério da } \\
\text { Saúde }\end{array}$ & $\begin{array}{l}\text { A anemia por deficiência de } \\
\text { ferro é considerada um grave } \\
\text { problema de saúde pública, é } \\
\text { considerada a carência } \\
\text { nutricional de maior magnitude. }\end{array}$ & BVS & 2013 \\
\hline 2 & $\begin{array}{l}\text { Anemia ferropriva infantil no } \\
\text { Brasil: uma revisão sistemática } \\
\text { de literatura. }\end{array}$ & $\begin{array}{l}\text { LEITÃO, Waldeir de } \\
\text { Sousa; OLIVEIRA, } \\
\text { Euzébio de }\end{array}$ & $\begin{array}{c}\text { fortificação com ferro em } \\
\text { alimentos utilizados na } \\
\text { alimentação infantil e programas } \\
\text { de educação alimentar para } \\
\text { melhor adequação do ferro total } \\
\text { e biodisponível. }\end{array}$ & $\begin{array}{l}\text { Google } \\
\text { Acadêmico }\end{array}$ & 2016 \\
\hline 3 & Iron Deficiency Anemia & DELOUGHERY TG. & $\begin{array}{l}\text { Iron replacement can be done } \\
\text { orally, or in patients in whom } \\
\text { oral iron is not effective or } \\
\text { contraindicated, with } \\
\text { intravenous iron. }\end{array}$ & PubMed & 2017 \\
\hline 4 & $\begin{array}{l}\text { Diagnóstico E Tratamento Da } \\
\text { Anemia Ferropriva. }\end{array}$ & $\begin{array}{l}\text { FREIRE, Sarah Torres. } \\
\text { et al. }\end{array}$ & $\begin{array}{l}\text { A partir das informações obtidas } \\
\text { neste estudo, } \\
\text { verifica-se que a anemia } \\
\text { ferropriva ainda é elevada nos } \\
\text { grupos mais vulneráveis, } \\
\text { crianças, mulheres em idade } \\
\text { reprodutiva e grávidas. }\end{array}$ & $\begin{array}{l}\text { Google } \\
\text { Acadêmico }\end{array}$ & 2020 \\
\hline 5 & $\begin{array}{l}\text { Fisiopatologia, diagnóstico e } \\
\text { tratamento da anemia } \\
\text { ferropriva: Uma revisão de } \\
\text { literatura. }\end{array}$ & BRITO, M. E. et al. & $\begin{array}{l}\text { Além disso, é visto que são } \\
\text { necessárias inúmeras maneiras } \\
\text { de prevenção e tratamento pois } \\
\text { trata-se de um problema de } \\
\text { saúde pública. }\end{array}$ & $\begin{array}{l}\text { Google } \\
\text { Acadêmico }\end{array}$ & 2021 \\
\hline
\end{tabular}

Fonte: Autores.

\section{Discussão}

A anemia ferropriva é causada principalmente pela perda aumentada de sangue ou da demanda aumentada por ferro, que pode ocorrer nas mulheres quando estão no período da gestação e menstruação, nos homens e nas mulheres após a menopausa (Cançado \& Chiattone, 2010; Santis, 2019). Segundo Camaschella (2019) a AF pode ocorrer por conta do aumento dos requisitos de ferro (Bebês, pré-escolares, adolescentes), baixa ingestão de ferro (vegetarianos, veganos), diminuição da absorção intestinal de ferro que pode ocorrer em casos de gastrite atrófica autoimune e infecção por Helicobacter pylori. Júnior et al., (2016) cita que a anemia ferropriva resulta da interação de múltiplos fatores etiológicos como: ingestão deficiente de ferro, devido ao baixo consumo de alimentos de origem animal, baixo nível socioeconômico, as precárias condições de saneamento e a alta prevalência de doenças infecto parasitarias, principalmente as que provocam perdas sanguíneas crônicas.

Segundo Maman (2020) a anemia ferropriva pode ocorrer por conta de: perda sanguínea (como nas neoplasias, hemorragia por trauma, sangramento gastrointestinal oculto), demanda aumentada (como na perda de ferro durante a gestação, o parto e a lactação), ingestão insuficiente e absorção inadequada. E apresenta sinais e sintomas como: dificuldade de aprendizagem nas crianças, cansaço generalizado, palidez de pele e mucosas, menor disposição para o trabalho e falta de apetite (Brasil, 2015).

Camaschella (2019) afirma que os sinais e sintomas da anemia por deficiência de ferro são negligenciados. A fadiga é um sinal importante, porém não é específico, síndrome das pernas inquietas, boca seca, queilite, glossite atrófica, teias faríngeas de Plummer-Vinson e queda de cabelo também são considerados sinais e sintomas. Segundo André et al. (2018) e Yamagishi et al. (2017) um dos maiores problemas de saúde pública no mundo é a anemia por deficiência de ferro, que se destaca como a 
principal carência nutricional, essa carência ocorre em três estágios:

$1^{\circ}$ depleção do estoque de ferro, que afeta os depósitos podendo evoluir para uma grave carência de ferro;

$2^{\circ}$ eritropoese ineficaz, onde os níveis de ferritina e o de hemoglobina apresentam-se diminuídos;

$3^{\circ}$ anemia ferropriva, sendo o estágio mais grave determinado pela queda dos níveis de hemoglobina.

Seu diagnóstico é feito por meio de exames laboratoriais como o hemograma, que mostrará uma diminuição nos níveis de hemoglobina, com presença de anemia microcítica e hipocrômica, uma baixa nos índices hematimétricos (VCM, HCM e CHCM), aumentado do RDW e diminuição do ferro sérico (Freire, et al., 2020). São feitos também a dosagem de ferro sérico, transferrina e saturação da transferrina, deficiência de ferro armazenado (ferritina) (Grotto, 2010; Svarch Guerchicoff, 2015; Machado et al., 2016). Segundo Nunes \& Barone (2013), através do esfregaço sanguíneo podemos observar as alterações do eritrócito como: coloração, tamanho ou estrutura e forma, porém para tal observação é necessário o auxílio de um microscópio convencional.

Gurmini et al (2018) afirma que para diagnóstico e para a profilaxia da anemia são preferencialmente o hemograma completo, contagem de reticulócitos e ferritina (usado como marcador da fase de depleção). Já Galeigo, et al (2014) cita que o processo infeccioso ou inflamatório concomitante é indicado pela dosagem de proteína C Reativa e o diagnóstico de anemia ferropriva pode ser confirmado no caso de PCR negativo associado a microcitose, hipocromia e ferritina sérica menor que 12 $\mathrm{ng} / \mathrm{mL}$. Nos casos de anemia ferropriva deve-se realizar tratamento nutricional através fármacos, de suplementos nutricionais ou através de alimentos ricos em ferro, com intuito de corrigir a deficiência do nutriente e restaurar os níveis de hemoglobina. O tratamento nutricional é eficaz e ocorre por via oral, envolvendo a ingestão de alimentos fontes de ferro (FREIRE, et al., 2020).

Segundo DeLoughery TG (2017) o ferro pode ser reposto por via oral, ou ferro intravenoso no caso de pacientes que ferro oral é contraindicado ou não é eficaz. Já Brito (2021) afirma que em alguns casos a terapia por via oral não é suficiente para normalizar a hemoglobina ou restaurar os depósitos normal de ferro, por tanto a aplicação por via parenteral é uma opção que deve ser considerada pois pode ser considerada segura, eficaz e efetiva. Esse tratamento é realizado com injeção intravenosa lenta ou infusão, comumente é 200mg por infusão e os principais medicamentos são: carboximaltose férrica, ferro gluconato, ferro sacarato e ferro dextran.

Além dos devidos tratamentos as principais prevenções que devem ser tomadas são: suplementação profilática com ferro e ácido fólico (na gestação), amamentação na primeira hora de vida e aleitamento materno exclusivo até os seis meses de vida, alimentação balanceada e saudável com ingestão de ferro, a ingestão de alimentos que contenham farinhas enriquecidas com ferro e ácido fólico, suplementação profilática de ferro para crianças prematuras e que nasceram com baixo peso (BRASIL, 2013).

Leitão e Oliveira (2016) alega que para combater e prevenir a anemia ferropriva deve ocorrer estratégias importantes como:

- $\quad$ Suplementação medicamentosa profilática

- $\quad$ Educação alimentar, fortificação alimentar e melhoria da qualidade da dieta oferecida;

- Incentivo ao aleitamento materno exclusivo nos primeiros seis meses de vida, que é de fundamental importância;

- $\quad$ Administração oral de ferro na forma ferrosa e associado ao tratamento medicamentoso, deve-se orientar o consumo de alimentos com quantidade e biodisponibilidade elevadas de ferro.

\section{Considerações Finais}

Mediante a pesquisa realizada foi possível concluir que a anemia ferropriva é considerada a mais comum das anemias, podendo ser citada como um sério problema de Saúde Pública que acomete principalmente mulheres e crianças, com destaque 
principal nos países em desenvolvimento.

- $\quad$ É causada por diversos fatores que podem ser internos e externos, onde qualquer sintoma sendo ele específico ou não precisa ser analisado;

- $\quad$ Seu diagnostico deve ser detalhado;

- Seu tratamento deve ser completo e acompanhado por um profissional da área, para que não ocorra novos problemas relacionados a essa patogenia. Se faz mais que necessário que modos de prevenção sejam passados para a sociedade e assim evitar que os indivíduos possam sofrer com essa condição.

\section{Referências}

Abbas, A.K. et al. Robbins Patologia Básica9: Robbins Patologia Básica. Elsevier Health Sciences. (9a ed.).

André, H. P. et al. Indicadores De Insegurança Alimentar E Nutricional Associados À Anemia Ferropriva Em Crianças Brasileiras: Uma Revisão Sistemática. Ciência \& Saúde Coletiva, 23, 1159-1167

Antunes, A. S., \& Canziani, M. E. F. Hepcidina: Um Importante Regulador Do Metabolismo De Ferro Na Doença Renal Crônica. J Bras Nefrol, 38 , 351-5

Azevedo, M. R. A. Hematologia Básica: Fisiopatologia E Diagnóstico Laboratorial. Thieme Revinter Publicações Ltda, 2019.

Baynes, J., \& Dominiczak, M. H. Bioquímica Médica + Studentconsult. (4a ed.), Elsevier España, 2015. 656 P.

Bortolini, Gisele A., \& Fisberg, M. Orientação Nutricional Do Paciente Com Deficiência De Ferro. Revista Brasileira De Hematologia E Hemoterapia, 32, 105113.

Brasil. Ministério Da Saúde. Agência Nacional De Vigilância Sanitária Anemia Por Deficiência De Ferro. Saúde E Economia, 5, 1-4.

Brasil. Ministério Da Saúde. Anemia. Brasília, 2015. <Http://Bvsms.Saude.Gov.Br/Dicas-Em-Saude/431-Anemia> .

Brasil. Ministério Da Saúde. Programa Nacional De Suplementação De Ferro: Manual De Condutas Gerais. 2013.

Brito, M. E. et al. Fisiopatologia, Diagnóstico E Tratamento Da Anemia Ferropriva: Uma Revisão De Literatura. Revista De Casos E Consultoria, 12, E23523, 25 .

Camaschella, C. Iron Deficiency. Blood, 133, 30-39.

Camaschella, C. Iron-Deficiency Anemia. New England Journal Of Medicine, 372, 1832-1843.

Cançado, R. D., \& Chiattone, C. S. Anemia Ferropênica No Adulto: Causas, Diagnóstico E Tratamento. Revista Brasileira De Hematologia E Hemoterapia, 32, 240-246, 2010.

Cançado, R. D., Lobo, C., \& Friedrich, J. R. Tratamento Da Anemia Ferropriva Com Ferro Por Via Oral. Revista Brasileira De Hematologia E Hemoterapia, $32,114-120$

Castro, T. G. et al. Anemia E Deficiência De Ferro Em Pré-Escolares Da Amazônia Ocidental Brasileira: Prevalência E Fatores Associados. Cadernos De Saúde Pública, 27, 131-142

Deloughery Tg. Iron Deficiency Anemia. Med Clin North Am.

Filho, L. F., \& Barros, E. Medicina Interna Na Prática Clínica. Artmed Editora. 1076 P.

Fisberg, M. et al. Consenso Sobre Anemia Ferropriva: Mais Que Uma Doença, Uma Urgência Médica. Sociedade Brasileira De Pediatria, 1-13

Freire, S. T. et al. Diagnóstico E Tratamento Da Anemia Ferropriva. Referências Em Saúde Da Faculdade Estácio De Sá De Goiás-Rrs-Fesgo, 3 ,

Galeigo, F. P. et al. Anemia E Senilidade. 2014

Grotto, H. Zw. Diagnóstico Laboratorial Da Deficiência De Ferro. Revista Brasileira De Hematologia E Hemoterapia, 32 , 22-28

Gurmini, J. et al. Consenso Sobre Anemia Ferropriva: Mais Que Uma Doença, Uma Urgência Médica!. Sbp, 2018.

Jameson, J. L. et al. Medicina Interna De Harrison. (20a ed.), Mcgraw Hill Brasil, 2.

Jordão, R. E. et al. Prevalência De Anemia Ferropriva No Brasil: Uma Revisão Sistemática. Revista Paulista De Pediatria, 27, 90-98

Júnior, J. G. De A. S. et al. Anemia Associada Às Parasitoses Intestinais De Pacientes Atendidos Em Um Laboratório De Análises Clínicas No Município De Juazeiro Do Norte-Ce. Revista Interfaces: Saúde, Humanas E Tecnologia, 3

Justino, L. De C. et al. Prevalência De Anemia Em Crianças Nos Últimos 10 Anos No Brasil. 2017. 
Research, Society and Development, v. 10, n. 9, e28310917894, 2021

(CC BY 4.0) | ISSN 2525-3409 | DOI: http://dx.doi.org/10.33448/rsd-v10i9.17894

Kauffman, P., \& Helito, A. S. De. Saúde: Entendendo As Doenças, A Enciclopédia Médica Da Família. Nobel, 2007. 718 P.

Leitão, W. De S., \& Oliveira, E. De. Anemia Ferropriva Infantil No Brasil: Uma Revisão Sistemática De Literatura. Revista Científica Multidisciplinar Núcleo De Conhecimento, 5, 24-36

Lemos, A. Dos R. et al. A Hepcidina Como Parâmetro Bioquímico Na Avaliação Da Anemia Por Deficiência De Ferro. Revista Da Associação Médica Brasileira, $56,596-599$

Lopes, D. L. et al. Aspectos Clinicos Pertinentes Na Anemia Ferropriva Em Crianças. Mostra Científica Em Biomedicina, 3, 2

Machado, G. S. et al. Abordagem Dos Principais Aspectos Relacionados À Anemia Ferropriva. Revista Conexão Eletrônica, 13, 1-6

Maman, M. J. C. De. Anemia Ferropriva. 2020.

Mendonça, C. R. De O. Anemia Por Deficiência De Ferro Em Idosos: Uma Revisão. 2018.

Nunes, D. C., \& Barone, A. Utilização De Marcadores Hematológicos Para Diferenciação De Anemia Ferropriva De Anemia De Doença Crônica. Atas De Ciências Da Saúde (Issn 2448-3753), 1

Nunes, M. P. Metabolismo Do Ferro E O Impacto Da Anemia Ferropriva À Saúde Humana. 2018.

Peixoto, A. L. Avaliação Nutricional: Do Diagnóstico À Prescrição. A.S. Sistemas, 2014. 254 P.

Pereira, L. P. M. O Papel Da Helicobacter Pylori Na Anemia Ferropénica. 2018. Tese De Doutorado.

Rodrigues, V. C. et al. Deficiência De Ferro, Prevalência De Anemia E Fatores Associados Em Crianças De Creches Públicas Do Oeste Do Paraná, Brasil. Revista De Nutrição, 24, 407-420

Santis, G. C. De. Anemia: Definição, Epidemiologia, Fisiopatologia, Classificação E Tratamento. Medicina (Ribeirao Preto), 52, 239-251

Sato, E. I. et al. Atualização Terapêutica De Felício Cintra Do Prado, Jairo De Almeida Ramos, José Ribeiro Do Valle: Diagnóstico E Tratamento. 26. Ed. São Paulo: Artes Médicas, 2018. 2180 P

Siqueira, K. N., \& Lenhard-Vidal, A. Perfil Epidemiológico Da Anemia Ferropriva Em Pacientes Atendidos Em Um Laboratório Clínico De Guarapuava-Pr. Tcc Biomedicina, 2019.

South-Paul, J. E. et al. Current: Medicina De Família E Comunidade (Lange): Diagnóstico E Tratamento. (3a ed.), Amgh Editora, 2014. 758 P.

Svarch Guerchicoff, E. Anemia Por Deficiencia De Hierro En El Lactante. Revista Cubana De Pediatria, 87, 395-398

Yamagishi, J. A. et al. Anemia Ferropriva. Revista Ciêntifica Da Faculdade De Educação E Meio Ambiente, 8, 99-110 